\title{
Tyrosine Hydroxylase Immunoreactivity in the Rhesus Monkey Retina Reveals Synapses from Bipolar Cells to Dopaminergic Amacrine Cells
}

\author{
Jan N. Hokoc and Andrew P. Mariani \\ Laboratory of Neurophysiology, National Institute of Neurological and Communicative Disorders and Stroke, National \\ Institutes of Health, Bethesda, Maryland 20892
}

The synaptic organization of dopamine-containing amacrine cells in the rhesus monkey retina was studied using immunohistochemistry of tyrosine hydroxylase (TH), the rate-limiting enzyme in the catecholamine synthetic pathway. Cell bodies of the TH-containing neurons were primarily in the innermost tier of the inner nuclear layer. Their synaptic processes, confined to the outermost stratum of the inner plexiform layer, contained mostly small, clear vesicles and were presynaptic to unlabeled amacrine cell processes and cell bodies at junctions that were symmetrical. Synapses onto the TH-immunoreactive neurons were from bipolar cell axon terminals, nonimmunoreactive amacrine cell processes, and other TH-containing amacrine cells in a decreasing order of predominance. The bipolar cells were presynaptic to the THcontaining neuronal processes at ribbon synapses. The size, structure, and position of the bipolar cell axon terminals, which, like the TH-reactive processes, were narrowly confined to the outermost stratum of the inner plexiform layer, indicate that they are the recently described giant bistratified bipolar cells. The identification of this bipolar cell input now provides evidence for a pathway from the outer plexiform layer to dopaminergic amacrine cells in the inner plexiform layer via a type of cone bipolar cell.

Since the initial description of catecholaminergic cells in the retina by Malmfors (1963), using the formaldehyde vapor method of Falck and Hillarp (Falck et al., 1962), the blue-green fluorescing dopaminergic neurons have been demonstrated repeatedly in a variety of vertebrate species. Anatomically, dopamine has been localized to a type of amacrine cell with somata in the innermost row of the inner nuclear layer and processes ramifying in the outermost stratum of the inner plexiform layer; and, in some species, it has been localized to a type of interplexiform cell as well (see review by Ehinger, 1982) by means of the classical aldehyde-induced fluorescent methods and, more recently, by immunohistochemistry. In mammals, the release of dopamine (Kramer, 1971) and the activitation of tyrosine hydroxylase (TH) (Iuvone et al., 1978), the rate-limiting enzyme in its synthetic pathway, are both effected by light. Postsynap-

\footnotetext{
Received Oct. 14, 1986; revised Mar. 19, 1987; accepted Mar. 19, 1987.

J.N.H. was a Fogarty International Research Fellow (F05 TW03584) sponsored at the NIH by R. Nelson and is on leave from the Federal University of Rio de Janeiro.

Correspondence should be addressed to A. P. Mariani, Bldg. 36, Rm. 2C-23, $\mathrm{NIH}$, Bethesda, MD 20892.

0270-6474/87/092785-09\$02.00/0
}

tically, it has been suggested that dopamine regulates the activity of other retinal neurons. The mechanism in fish retina is probably the uncoupling of gap junctions (Teranishi et al., 1983) and may involve a second messenger system (Watling et al., 1979). A similar uncoupling of gap junctions in the inner plexiform layer has been suggested for mammalian retinas (Mariani et al., 1984). Thus, while much is known about the localization, distribution, release, and effects of dopamine in the retina, little is known concerning its synaptic input from other types of retinal neurons. Such data would provide information regarding the nature of the physiological input to this well-studied transmitter phenotype.

Previous electron-microscopic studies of the synaptic organization of dopamine-containing neurons have relied on the uptake of neurotoxic-amine analogs such as 6-hydroxydopamine and 5,6-dihydroxytryptamine (Dowling and Ehinger, 1975, 1978; Akagi et al., 1980; Dowling et al., 1980; Holmgren, 1982) to induce degenerative changes in the cells and so label them for analysis or for electron-microscopic autoradiography of ${ }^{3} \mathrm{H}$ dopamine uptake (Frederick et al., 1982; Holmgren-Taylor, 1982; Pourcho, 1982). On the basis of these studies, dopaminergic amacrine cells are said to be "interamacrine," as they have been found only pre- and postsynaptic to other amacrine cells. This finding suggests that the regulation of the dopaminergic amacrine cells is carried out by other amacrine cells, and is thus at least one synapse removed from the bipolar cells that convey light information transduced by photoreceptors from the outer plexiform layer to the inner plexiform layer.

However, we have now used a different approach to study the synaptic organization of the dopaminergic amacrine cells in the rhesus monkey retina. Electron-microscopic immunohistochemistry of antibodies directed against $\mathrm{TH}$, a sensitive and reliable method successfully employed in the brain (Pickel et al., 1975) as a marker for catecholaminergic neurons, has now been used in the retina. The results reveal the existence of synaptic input to the dopaminergic amacrine cells from the axon terminals of a specific, recently identified type of cone bipolar cell. This new finding provides evidence for a light-mediated pathway from the outer plexiform layer to the dopaminergic amacrine cells in the inner plexiform layer, and significantly alters current conceptions of the dopaminergic amacrine cell type as "interamacrine."

\section{Materials and Methods}

Eyes were obtained from 6 juvenile (3-5 year-old) rhesus monkeys, Macaca mulatta, that were deeply anesthetized with pentobarbital sodium. The globes were injected with $3 \mathrm{ml}$ of fixative through a syringe 


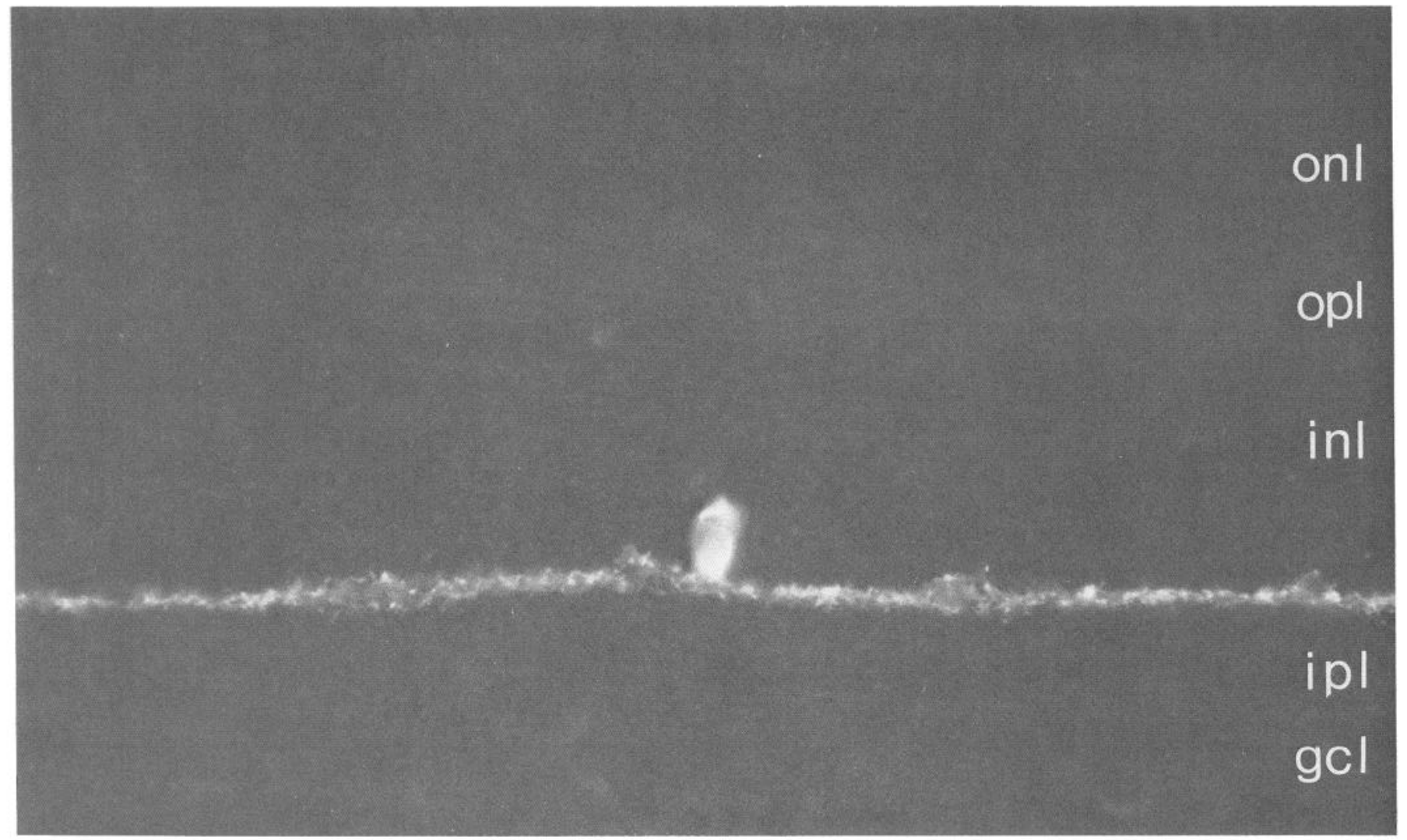

Figure 1. Light micrograph of tyrosine hydroxylase $(\mathrm{TH})$ immunoreactivity in the central rhesus monkey retina, visualized by the indirect immunofluorescent method with a fluorescein-conjugated secondary antibody. The immunoreactive cell body is in the innermost row of the inner nuclear layer $(\mathrm{inl})$ and TH-containing processes arborize in the outermost stratum of the inner plexiform layer (ipl). onl, Outer nuclear layer; opl, outer plexiform layer; $g c l$, ganglion cell layer. $\times 595$.

equipped with a 19-gauge hypodermic needle, and a second needle allowed outflow of vitreous and other intraocular fluids. The eyes were subsequently enucleated, bisected with a razor, and the posterior pole immersed in fixative. For light microscopy, retinas were fixed in $4 \%$ formaldehyde in $0.075 \mathrm{M}$ phosphate buffer. A $1 \times 1 \mathrm{~cm}$ square centered on the fovea was dissected from the eyecup, and frozen sections 15-20 $\mu \mathrm{m}$ thick were cut in the radial plane. The sections were mounted on gelatinized slides and incubated overnight at $4^{\circ} \mathrm{C}$ with highly specific antisera raised against $\mathrm{TH}$. These antisera were against the tryptic digest form of TH; they were isolated and purified from bovine adrenal medulla, and raised in rabbits. They were purchased from Eugene Tech Int. (Allendale, NJ). Previous studies with these antisera demonstrated that they reacted with the morphologically identical populations of neurons that displayed aldehyde-induced catecholamine fluorescence in other tissues of the central nervous system (Mariani et al., 1986). The antisera were reconstituted and diluted according to specifications provided by the manufacturer. The primary reaction was visualized by fluorescein isothiocyanate (FITC)-conjugated goat anti-rabbit secondary antibodies, at a dilution of 1:30, by the indirect immunofluorescent method (Coons, 1958). Controls consisted of sections incubated with normal rabbit serum, at a dilution of 1:500, instead of with the primary antisera, or with the primary antisera excluded from the incubation, while otherwise processed the same way and in parallel with the TH antisera. No immunoreactivity was noted in either of the controls. Triton $\mathrm{X}-100,0.25 \% \mathrm{vol} / \mathrm{vol}$, was used in the solutions to enhance the permeability of the reagents. The sections were mounted in a medium that retards fluorescent fading (Gilok and Sedat, 1982). Three retinas from 3 monkeys were surveyed for immunoreactivity by light microscopy. We chose this method because of the superior resolution of fluorescence for light microscopy.

Antisera against dopamine beta-hydroxylase (DBH) and phenylethanolamine- $N$-methyltransferase (PNMT), enzymes that synthesize norepinephrine and epinephrine, respectively (Eugene Tech), were applied to sections of the 3 retinas in an identical fashion to that used for the $\mathrm{TH}$ antisera, but no immunoreactivity was found.

For electron microscopy, retinas were fixed as outlined above, but with $0.1 \%$ glutaraldehyde added to the fixative. The tissue was infiltrated in $30 \%$ sucrose in buffer and rapidly frozen in 2-methylbutane cooled by liquid $\mathrm{N}_{2}$ and thawed in buffer to permit penetration of antibodies. Approximately $1 / 4-1 / 2 \mathrm{~mm}$ sections were cut by hand with a razor, and the strips incubated as above with the $\mathrm{TH}$ antisera with the omission of Triton X-100. The primary reaction was visualized by the peroxidaseantiperoxidase (PAP) method (Sternberger, 1986). Goat anti-rabbit IgG, $1: 50$, and rabbit PAP, $1: 100$, followed by diaminobenzidine as a chromagen, were used in this procedure. Controls were as for light microscopy. The slices were subsequently osmicated, stained en bloc with uranyl acetate, rapidly dehydrated in a graded series of ethanol, cleared in propylene oxide, infiltrated, and embedded in Epon. Initially, thin sections were examined without further staining in the electron microscope. Later they were doubly stained with an ethanolic solution of uranyl acetate (Gibbons and Grimstone, 1960) and lead citrate (Venable and Coggeshall, 1965). The electron microscopy was based on retinal tissue from 3 monkeys.

\section{Results}

\section{Light microscopy}

Indirect immunofluorescence revealed TH-containing neuronal cell bodies, primarily in the innermost row of the inner nuclear layer (Fig. 1). A few were found slightly more sclerad in the inner nuclear layer, in the middle of the inner plexiform layer, and in the ganglion cell layer. The TH-immunoreactive somata were $10-15 \mu \mathrm{m}$ in diameter and consisted of a rim of intensely fluorescent cytoplasm surrounding a $9-\mu \mathrm{m}$-diameter nonreactive nucleus. TH-immunoreactive processes arborized almost exclusively in the outermost stratum of the inner plexiform 


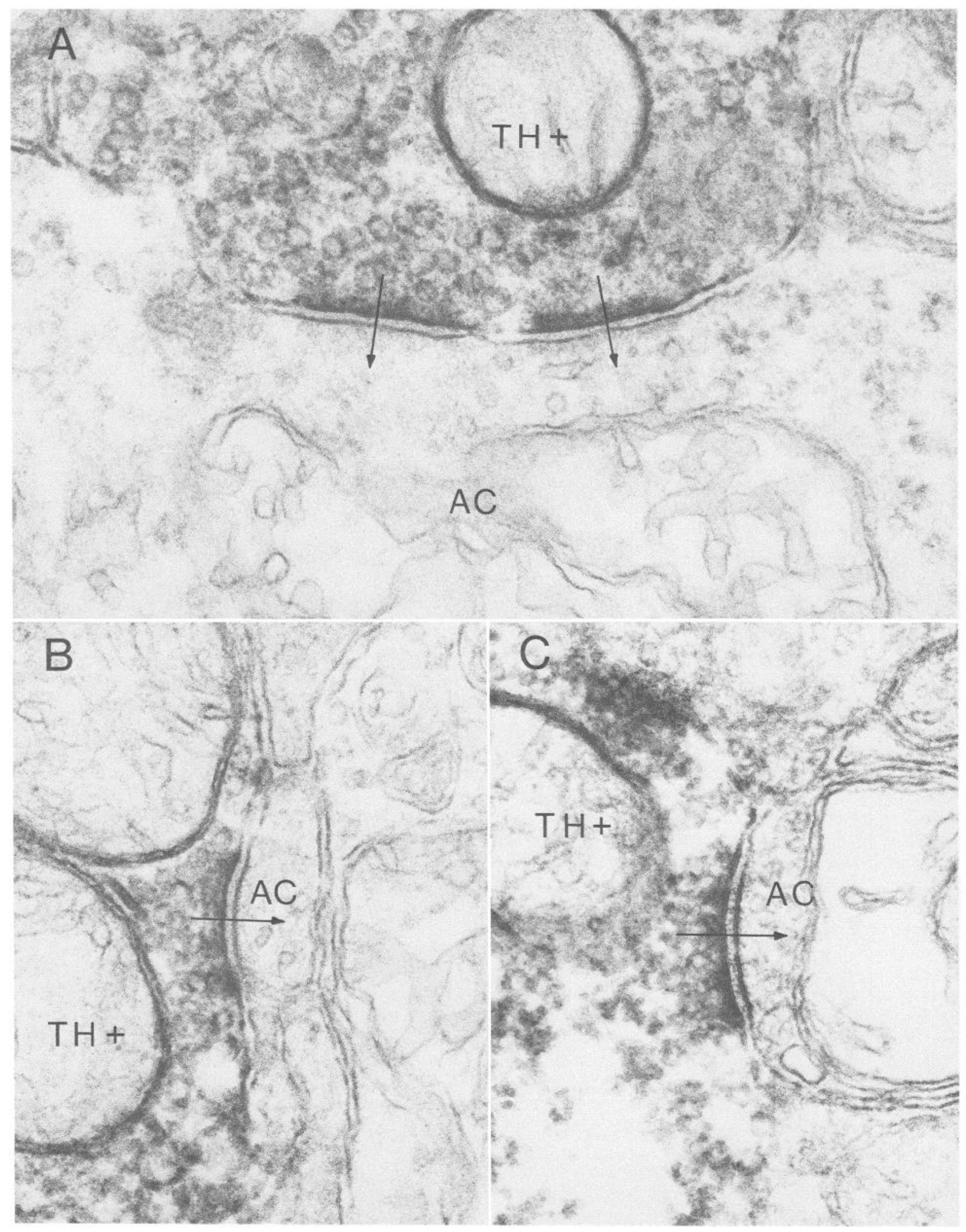

Figure 2. A, Electron micrograph of a TH-immunoreactive cell process $(T H+)$ that makes 2 synapses onto an unlabeled amacrine cell $(A C)$ in the inner plexiform layer. In this and subsequent figures, arrows indicate the position and the polarity of the synapses. $\times 113,600$. $B$ and $C$, THcontaining amacrine cell processes $(T H+)$ are presynaptic to unlabeled amacrine cell processes $(A C)$. The synapses, classified as of the symmetrical type, display a cluster of small, clear vesicles with a thickening at the cytoplasmic aspect of the presynaptic processes. Two smoothly parallel membranes are separated by a widened intercellular cleft. An electron-opaque material is present in the synaptic cleft. No apparent postsynaptic thickening is present at these junctions. $\times 113,600$. 
layer, directly below the cell bodies of origin in the inner nuclear layer (Fig. 1). Here they formed a narrow, 6.2- $\mu$ m-deep, but solid and strongly immunoreactive lamina. They were sometimes observed deeper in the inner plexiform layer, spreading laterally in the centralmost stratum. Very fine $1 / 2-\mu \mathrm{m}$-diameter varicose TH-immunoreactive processes, which originated either from the $\mathrm{TH}$-containing cell bodies or from their processes in the inner plexiform layer, often extended through the inner nuclear layer. Here they either ended, coursed back to the inner plexiform layer, or reached the outer plexiform layer. Despite the fact that these processes were often observed to enter the outer plexiform layer, they were invariably found to end as a small swelling, never arborizing. This localization of TH immunoreactivity is identical to that reported in the macaque retina by others for the aldehyde-induced fluorophore of dopamine (Laties and Jacobowitz, 1966; Mariani et al., 1984) and to results of immunohistochemistry with other TH antisera (Nguyen-Legros et al., 1984).

Although PNMT-immunoreactive amacrine cells have been described in the rat retina (Hadjiconstantinou et al., 1984), these likely epinephrinergic neurons are distinct in their morphology from the classically described dopaminergic neurons, and they arborize in a different stratum of the inner plexiform layer. Furthermore, both DBH and PNMT were negative for immunoreactivity in the rhesus monkey retina, as noted in Materials and Methods, indicating that catecholamine synthesis in the rhesus retina does not proceed beyond dopamine, but we cannot yet exclude the possibility of false negatives for DBH and PNMT. Taken together, however, these results can be interpreted as evidence that the neurons identified here by $\mathrm{TH}$ immunoreactivity are the dopaminergic neurons.

\section{Electron microscopy}

In the electron microscope, $\mathrm{TH}$ immunoreactivity was identified by the electron opacity of the osmicated HRP reaction product. Our observations were confined to the immunoreactive processes located in the outermost stratum of the inner plexiform layer. The TH-containing elements in the center stratum of the inner plexiform layer were too infrequently encountered to provide a definite statement concerning their synaptic organization. The HRP reaction product was localized to profiles that contained mitochondria that excluded the HRP and to small, clear synaptic vesicles that aggregated at the membrane and formed conventional synapses. Owing to the characteristic cytological appearance of neuronal elements in the inner plexiform layer, the TH-immunoreactive processes could be identified as amacrine since they were presynaptic to other elements at conventional chemical synapses. In contrast, the synaptic endings of bipolar cells contain a synaptic ribbon as a presynaptic specialization, while the dendrites of ganglion cells that often possess numerous ribosomes are postsynaptic in the retina of this species (Dowling and Boycott, 1966).

The synapses formed by the TH-containing amacrine processes consisted of a widened intercellular cleft of $13.6 \mathrm{~nm}$ between 2 smoothly parallel membranes. The cleft contained a material of moderate electron opacity in its center, which was oriented parallel to the membranes. This often had the appearance of a fine, faint line bisecting the cleft. A row of 4-6 small, clear vesicles was in register with a 29 -nm-thick presynaptic density in the TH-immunoreactive process. There was no apparent postsynaptic thickcning at thesc junctions (Fig. 2, $A-C$ ). In spite of the opaque material lining the presynaptic site of the
TH-containing process, the absence of any postsynaptic membrane thickening or opacity categorizes these synapses as symmetrical (Colonnier, 1968) or as Gray's (1959) type II.

Synapses onto TH-immunoreactive processes, which accounted for $20 \%(n=32)$ of all synapses involving TH-containing profiles, were from bipolar ccll axon terminals, nonimmunoreactive amacrine cell processes, and other TH-positive amacrine cell profiles, in decreasing order of predominance.

The most common synaptic input was from bipolar cell axon terminals; these represented $53 \%(n=17)$ of the synaptic input to TH-immunoreactive processes in the inner plexiform layer. The bipolar cell synaptic endings were always presynaptic at ribbon synapses (Figs. $3, A-C ; 4, A, B$ ). These synapses were typically dyads where one of the postsynaptic processes was a TH-containing element and the other a nonimmunoreactive amacrine (Figs. 3, $A-C ; 4 B$ ). The size and arborization of the bipolar cell axon terminals, which, like the TH-immunoreactive processes, are narrowly confined to the outermost stratum of the inner plexiform layer directly adjacent to the inner nuclear layer (Fig. 3, $A, B$ ), suggest that they are the recently identified giant bistratified bipolar cells (Mariani, 1983). Two immunocytochemically labeled processes were not found to form both postsynaptic members of the dyad. Also, no ganglion cell dendrites were observed to be one of the postsynaptic elements of the dyad involving the TH-containing amacrine cell processes.

Unlabeled amacrine cell processes formed $31 \%(n=10)$ of the synapses onto the dopaminergic amacrines. The membranes at these synaptic contacts were smoothly parallel and separated by a widened intercellular cleft of $13 \mathrm{~nm}$. A small clustcr of 310 synaptic vesicles was present at the presynaptic side of the junction. The cytoplasmic aspect of the postsynaptic membrane was usually bordered by a $28-\mathrm{nm}$-thick opacity. Such junctions have the characteristics of asymmetrical (Colonnier, 1968) or Gray's type I (1959) synapses (Fig. 5B).

The least frequent synaptic input to $\mathrm{TH}$-immunoreactive amacrine cells was from other similarly labeled amacrine cells, and these junctions represented $16 \%(n=5)$ of the synaptic input to the dopaminergic neurons.

The synaptic output of the TH-containing amacrine cell processes accounted for $80 \%(n=116)$ of the synapses involving $\mathrm{TH}$-immunoreactive processes. These synapses were primarily onto nonimmunoreactive amacrine cells $(92 \%, n=107)$, and these contacts were either onto profiles in the inner plexiform layer or onto cell bodies in the inner nuclear layer (Fig. 2, $A-$ $C)$. Often, in a single plane of section, a single $\mathrm{TH}$-immunoreactive amacrine profile made 2 or 3 synaptic junctions onto one postsynaptic element (Fig. $2 A$ ). Much less frequently found were synapses onto bipolar cell axon terminals $(3 \%, n=4)$, and these occurred at regions where the TH-containing amacrine was also postsynaptic to the bipolar axon terminal, as described above (Fig. $5 A$ ). This synaptic arrangement has been termed a reciprocal synapse. The remainder of the THI-reactive synaptic output $(4 \%, n=5)$ was onto other $\mathrm{TH}$-labeled processes, as described above. No synaptic contacts involving TH-immunoreactive processes and ganglion cell dendrites were found. The results described above, derived from electron microscopy, have been replicated in 3 retinas from 3 different monkeys.

\section{Discussion}

Electron microscopy of TH immunoreactivity in the rhesus monkey retina has shown that the dopaminergic amacrine cells receive synaptic input from both bipolar cells and amacrine 


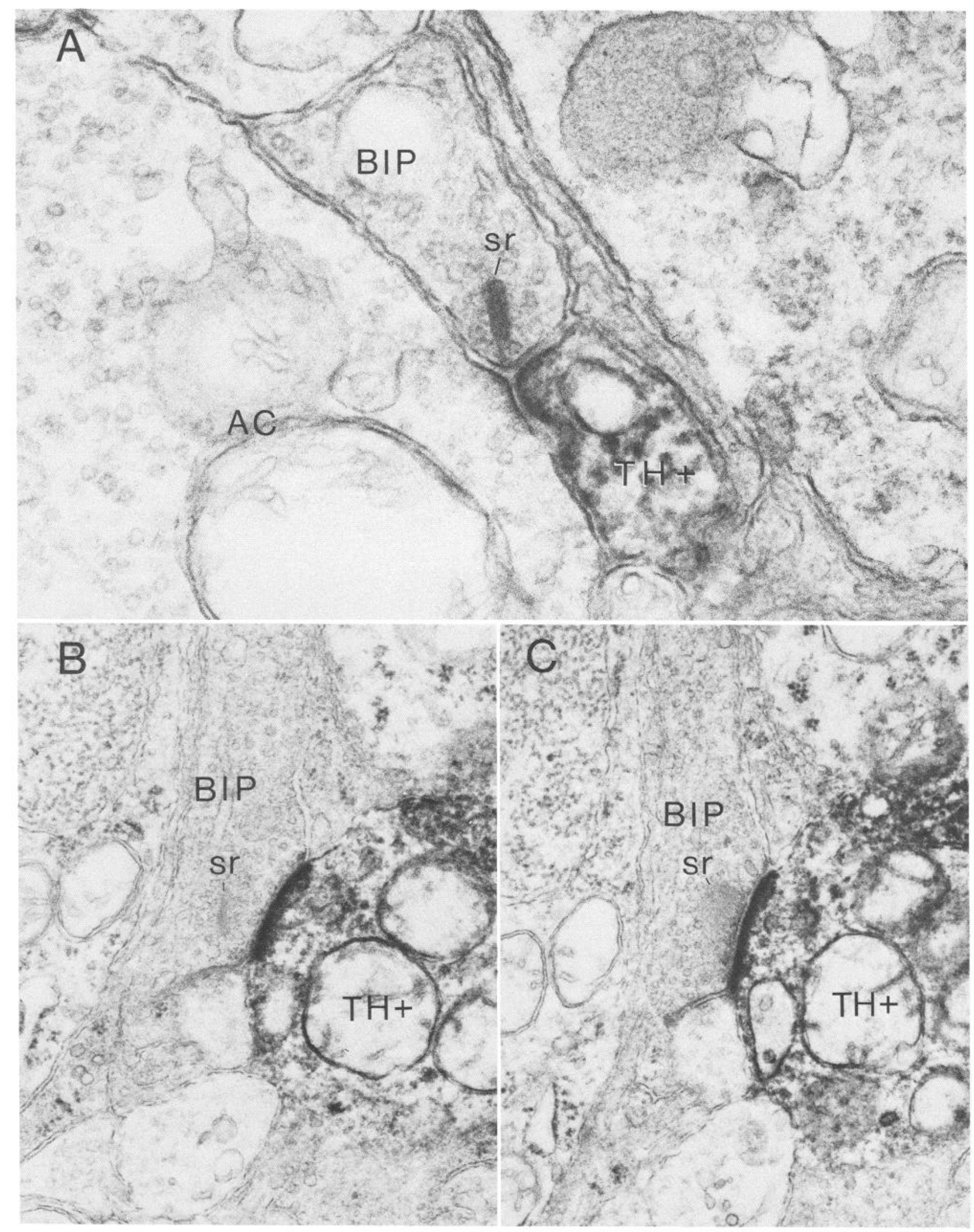

Figure 3. A, Bipolar cell axon terminal $(B I P)$ containing a synaptic ribbon $(s r)$ is presynaptic to the TH-immunoreactive amacrine cell process $(T H+)$. The other element of the postsynaptic dyad is a nonimmunoreactive amacrine cell $(A C) . \times 99,400$. B and $C$, Bipolar cell axon terminal $(B I P)$ in the outermost stratum of the inner plexiform layer is presynaptic at a dyad where the TH-containing amacrine cell process $(T H+)$ is one of the postsynaptic elements. The synaptic ribbon $(s r)$ is perpendicular to the plane of section in $B$ and tangential in $C$, the adjacent section. $\times 40,000$. 


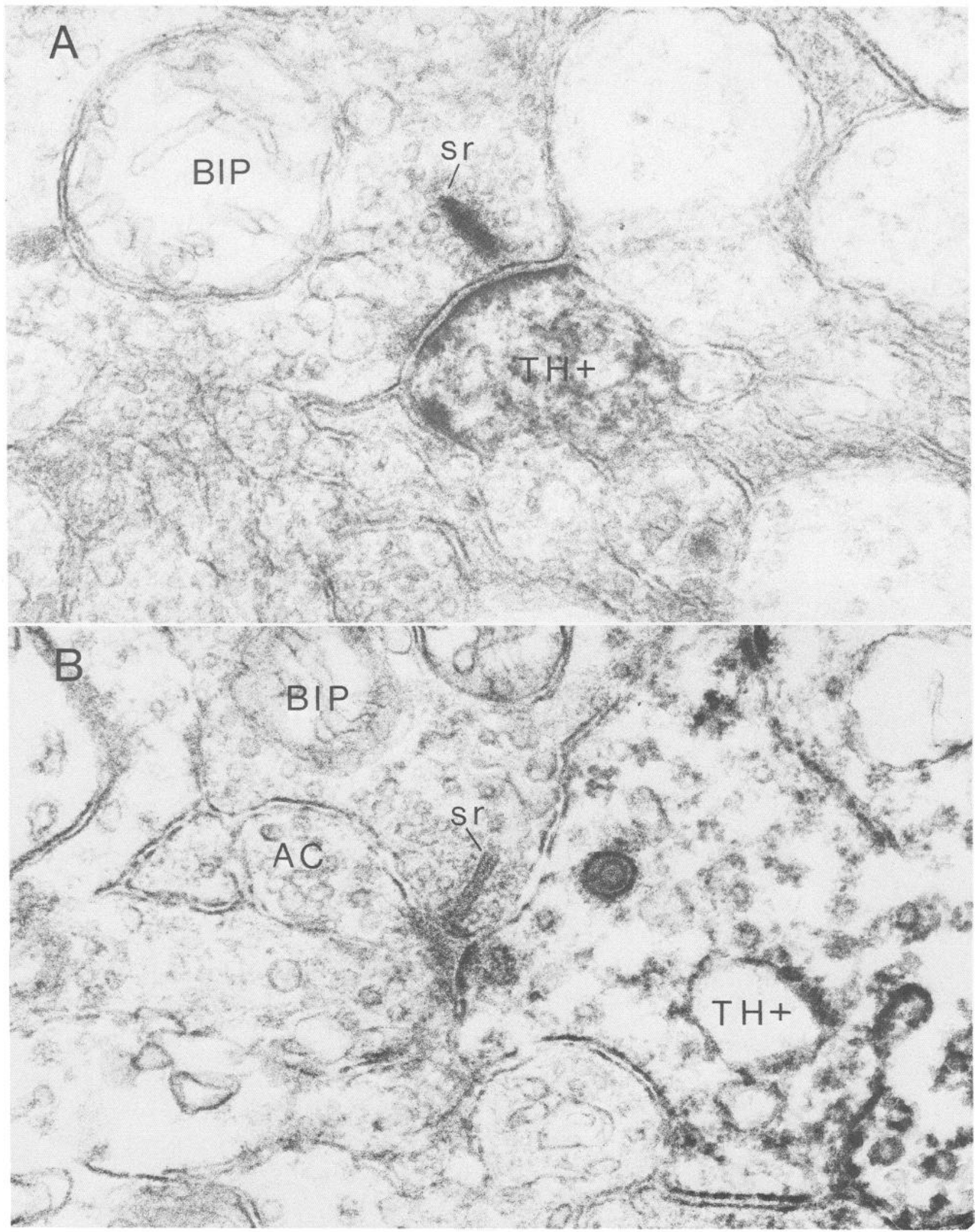

Figure 4. A, Bipolar cell axon terminal $(B I P)$ with a synaptic ribbon (sr) directly apposed to the presynaptic membrane has a single postsynaptic element, a TH-immunoreactive process $(T H+) . \times 99,400 . B$, TH-containing amacrine cell process $(T H+)$ is one member of the postsynaptic dyad at a bipolar cell $(B I P)$ ribbon synapse. $A C$, amacrine cell; $s r$, synaptic ribbon. $\times 99,400$. 


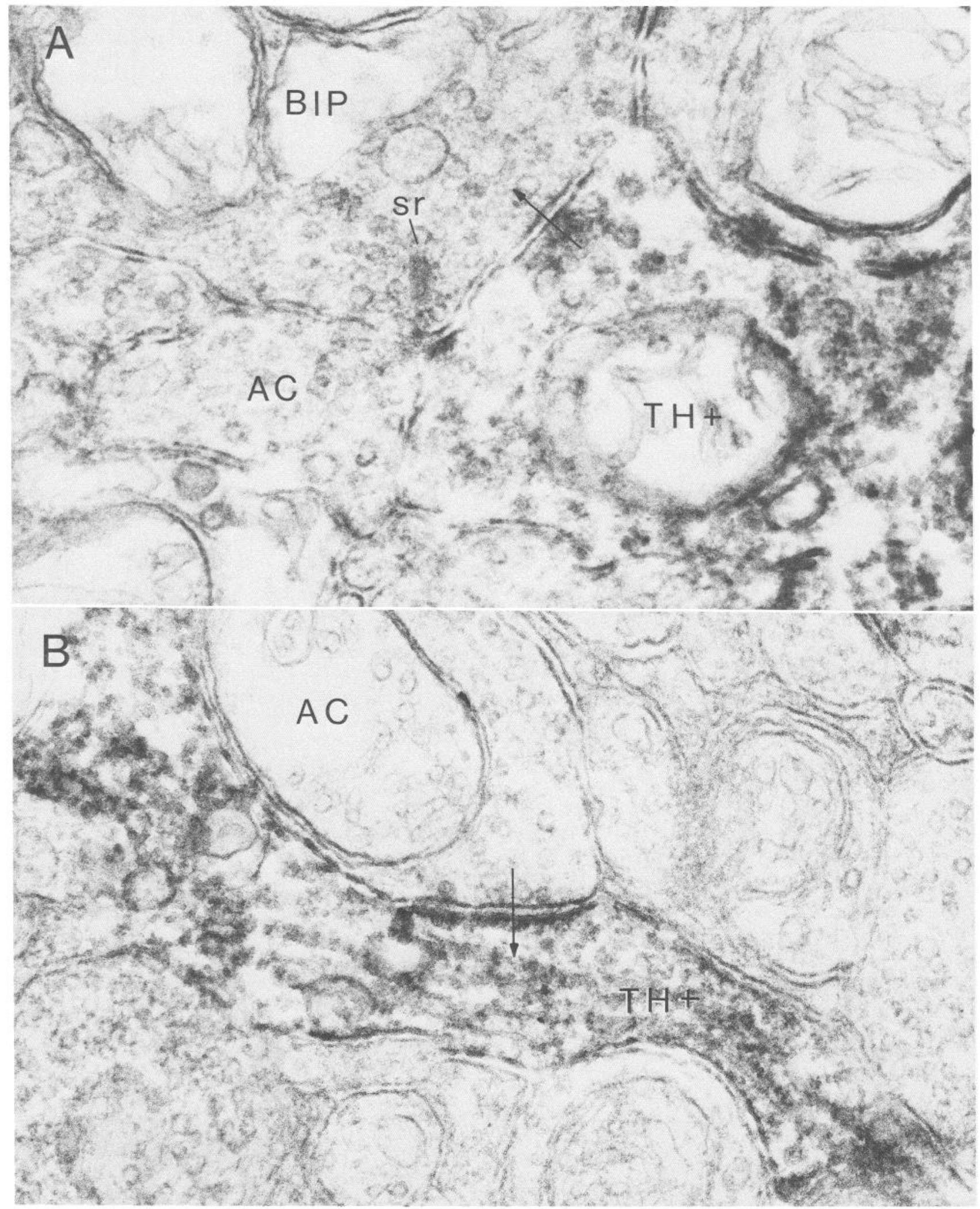

Figure 5. A, Reciprocal synapse of a TH-immunoreactive amacrine cell process $(T H+)$ with a bipolar cell axon terminal $(B I P)$. The synaptic ribbon $(s r)$ in the bipolar cell terminal indicates the point at which the bipolar cell is presynaptic to both labeled $(T H+)$ and unlabeled $(A C)$ amacrine cell processes. The arrow indicates the position and polarity of the reciprocal synapse from the TH+ process onto the bipolar cell. Here, there is a widened intercellular cleft, and an accumulation of synaptic vesicles at the presynaptic membrane. $\times 106,500$. $B$, Nonimmunoreactive amacrine cell process $(A C)$ is presynaptic to a TH-immunoreactive cell process $(T H+)$. A widened intercellular cleft separates the smoothly parallel membranes. The presynaptic side of the junction presents a row of synaptic vesicles, which indicates the polarity of the synapse (arrow). The thick opacity bordering the cytoplasmic side of the postsynaptic membrane of the $\mathrm{TH}+$ process distinguishes this synapse as asymmetrical. $\times 106,500$. 
cells, and, in turn are presynaptic to amacrine cells with very few synapses, in a reciprocal fashion, back onto the bipolar cell axon terminals. This study thus provides the first evidence for synaptic input to the dopaminergic neurons from bipolar cells in any retina. All previous investigations into the synaptic organization of the dopaminergic amacrine cells have described only amacrine synaptic input and output for this catecholaminergic phenotype, which was thus widely considered to be "interamacrine." Our data, however, provide evidence for a direct, light-mediated pathway from the outer plexiform layer involving a type of cone bipolar cell to the dopamine-containing amacrine cells in the inner plexiform layer.

In contrast to our use of antibodies generated against the ratelimiting enzyme in the catecholamine-synthetic pathway, other studics in which investigators used electron microscopy have depended on autoradiography of ${ }^{3} \mathrm{H}$-dopamine uptake or the lesioning of neurons with toxic amine analogs to induce degenerative changes in the cells and so label them for ultrastructural analysis (see the introduction). It seems likely, then, that the differences in methodology are likely to account for the differences in results. For example, while autoradiography generally allows excellent preservation of ultrastructure, it can be hampered by a lack of resolution associated with the precise identification of the source of radiation emitted, which is visualized as a silver grain. On the other hand, lesioning methods with the toxic analogs can suffer from problems associated with specificity, especially when indolamine analogs are used as uptake substrates for catecholamines; there are also problems arising from the application of the toxin, which is by injection into the vitreous, a tissue that forms a considerable barrier to the retina, preventing uniform labeling of the entire retina. Furthcrmorc, the duration and dose of the toxic amine analog must be empirically determined by the investigator to produce the optimal result, a somewhat subjective approach. Too little of the substance with regard to either time or dose may not produce distinctive degenerative changes, while too much would probably so completely destroy the cells that an ultrastructural analysis of synaptic organization would not be possible. In contrast, immunohistochemistry is not subject to the above deficiencies, but can, in certain instances, be limited by nonspecific crossreactivity, sensitivity to fixation, and lack of tissue penetration. However, these limitations were not encountered in the present study.

The giant bistratified bipolar cells that are presynaptic to the TH-containing amacrine processes have certain morphological features that may be related to their role in providing synaptic input to the dopaminergic amacrine cells. For example, the large, asymmetric axonal arborization of this bipolar cell type is the only one of the 7 bipolar cell types (Polyak, 1957; Boycott and Dowling, 1969; Mariani, 1981, 1983, 1984a, b) thus far identified in the monkey retina that arborizes precisely in the same stratum as do the processes of the TH-immunoreactive amacrine cells. In fact, this distinction was noted when these bipolar cells were first described (Mariani, 1983). The large size and characteristic asymmetry of this axonal arborization may be related to the low cellular density and nonregular organization of the dopaminergic neurons (Mariani et al., 1984). Furthermore, whereas all other bipolar cell types in monkey retina contact only 1-7 photoreceptors, the giant bistratified bipolar cells connect with as many as 20 cone pedicles in the outer plexiform layer. Thus, these bipolar cells, rather than being involved in selective chromatic or high-resolution pathways, are likely to sample light information over a large area and provide a convergent input to the low-density dopaminergic cells.

The identity of the nonimmunoreactive amacrine cell synapses onto the TH-containing cells remains to be elucidated. Other biochemical and pharmacological experiments suggest that GABAergic amacrine cells in the goldfish retina may provide this synaptic input (O'Brien and Dowling, 1985). In this regard, it should be noted that the nonimmunoreactive amacrine synapses onto the TH-containing cells are of the asymmetrical type, whereas the glutamate decarboxylase (GAD)-immunoreactive synapses onto non-GAD-containing amacrines are of the symmetrical type (Mariani and Caserta, 1986). This evidence does not favor direct synapses from the GABAergic phenotype to the dopaminergic phenotype in the monkey retina. However, the GABAergic amacrines were primarily pre- and postsynaptic to bipolar axon terminals, and it is possible that they represent the other postsynaptic member of a dyad involving the TH-reactive cells and provide an indirect modulation of the dopaminergic cells via the GABAergic feedback (reciprocal synapses) onto the bipolar cells.

The finding reported here that the dopaminergic neurons of the monkey retina have synaptic input from bipolar cells contrasts quite a bit with previous studies, indicated above, which have till now only shown synapses from other amacrine cells. While it is likely that methodological differences may underlie these differences in results, it seems important to establish whether our findings can be generalized to other species as well. It is clear that significant differences in neuronal morphology and organization exist even between species within a particular class of vertebrates (Mariani, 1985), but the dopaminergic amacrine cell phenotype is one that seems well-conserved among various species (Fhinger, 1982). Perhaps investigations in other species using $\mathrm{TH}$ immunohistochemistry will also reveal bipolar cell input to the dopaminergic neurons. In this respect, we should note that 2 other mammals, cat and rabbit, which are frequently used in anatomical and electrophysiological studies of the retina, also contain well-identified dopaminergic amacrine cells, and that both these species have a bipolar cell type similar to the monkey's giant bistratified bipolar cell (Famiglietti, 1981). Further investigations of the giant bistratified bipolar cells, focusing on their responses to light and on the identity of the neurotransmitter they release onto the dopaminergic amacrine cells, will undoubtedly provide more insight into the physiological regulation of synaptic input to the dopamine-containing amacrine cells of the primate retina.

\section{References}

Akagi, Y., M. Itoi, and Y. Sano (1980) Monoamine-containing neurons in the retina of monkey. Jpn. J. Ophthalmol. 24: 428-438.

Boycott, B. B., and J. E. Dowling (1969) Organization of the primate retina: Light microscopy. Phil. Trans. R. Soc. I ond. B 255: 109-176.

Colonnier, M. (1968) Synaptic patterns on different cell types in the different laminae of the cat visual cortex. An electron microscope study. Brain Res. 9: 268-287.

Coons, A. H. (1958) Fluorescent antibody methods. In General Cytochemical Methods, J. F. Danielli, ed., pp. 399-422, Academic, New York.

Dowling, J. E., and B. B. Boycott (1966) Organization of the primate retina: Electron microscopy. Proc. R. Soc. [Biol.] 166: 80-111.

Dowling, J. E., and B. Ehinger (1975) Synaptic organization of the amine-containing interplexiform cells of the goldfish and Cebus monkey retina. Science 188: 270-273.

Dowling, J. E., and B. Ehinger (1978) Synaptic contacts of dopaminergic neurons in the rabbit retina. J. Comp. Neurol. 180: 203-220. 
Dowling, J. E., B. Ehinger, and I. Floren (1980) Fluorescence and electron microscopical observations on the amine-accumulating neurons of the Cebus monkey retina. J. Comp. Neurol. 192: 665-685.

Ehinger, B. (1982) Neurolransmitler systems in the retina. Retina 2: 305-321.

Falck, B., N. A. Hillarp, G. Thieme, and A. Torp (1962) Fluorescence of catecholamines and related compounds condensed with formaldehyde. J. Histochem. Cytochem. 10: 348-354.

Famiglietti, E. V., Jr. (1981) Functional architecture of cone bipolar cells in mammalian retina. Vision Res. 21: 1559-1563.

Frederick, J. M., M. E. Rayborn, A. M. Laties, D. M. K. Lam, and J. G. Hollyfield (1982) Dopaminergic neurons in the human retina. J. Comp. Neurol. 210: 65-79.

Gibbons, I. R., and A. V. Grimstone (1960) On flagellar structure in certain flagellates. J. Biophys. Biochem. Cytol. 7: 697-716.

Gilok, H., and J. W. Sedat (1982) Fluorescence microscopy: Reduced photobleaching of rhodamine and fluorescein in protein conjugates by $n$-propyl gallate. Science $217:$ 1252-1255.

Gray, E. G. (1959) Axo-somatic and axo-dendritic synapses of the cerebral cortex: An electron microscope study. J. Anat. 93: 420-433.

Hadjiconstantinou, M., A. P. Mariani, P. Panula, T. H. Joh, and N. H. Neff (1984) Immunohistochemical evidence for epinephrine-containing retinal amacrine cells. Neuroscience 13: 547-551.

Holmgren, I. (1982) Synaptic organization of the dopaminergic neurons in the retina of the Cynomolgus monkey. Invest. Ophthalmol. Vis. Sci. 22: 8-24.

Holmgren-Taylor, I. (1982) Ultrastructure and synapses of the ${ }^{3} \mathrm{H}$ dopamine-accumulating neurons in the retina of the rabbit. Exp. Eye Res. 35: 555-572.

Iuvone, P. M., C. L. Galli, C. K. Garrison-Gund, and N. H. Neff (1978) Light stimulates tyrosine hydroxylase activity and dopamine synthesis in retinal amacrine neurons. Science 202: 901-902.

Kramer, S. G. (1971) Dopamine: A retinal neurotransmitter. I. retinal uptake, storage, and light-stimulated release of $\mathrm{H}^{3}$-dopamine in vivo. Invest. Ophthalmol. 10: 438-452.

Laties, A. M., and D. Jacobowitz (1966) A comparative study of the autonomic innervation of the eye in monkey, cat and rabbit. Anat. Rec. 156: 383-396.

Malmfors, T. (1963) Evidence of adrenergic neurons with synaptic terminals in the retina of rats demonstrated with fluorescence and electron microscopy. Acta Physiol. Scand. 58: 99-100.

Mariani, A. P. (1981) A difuse invaginating cone bipolar cell in primate retina. J. Comp. Neurol. 197: 661-671.
Mariani, A. P. (1983) Giant bistratified bipolar cells in the monkey retina. Anat. Rec. 206: 215-220.

Mariani, A. P. (1984a) Bipolar cells in the monkey retina selective for the cones likely to be blue sensitive. Nature 308: 184-186.

Mariani, A. P. (1984b) The neuronal organization of the outer plexiform layer of the primate retina. Int. Rev. Cytol. 86: 285-320.

Mariani, A. P. (1985) Multiaxonal horizontal cells in the retina of the tree shrew, Tupaia glis. J. Comp. Neurol. 233: 553-563.

Mariani, A. P., and M. T. Caserta (1986) Electron microscopy of glutamate decarboxylase (GAD) immunoreactivity in the inner plexiform layer of the rhesus monkey retina. J. Neurocytol. 15: 645-655.

Mariani, A. P., H. Kolb, and R. Nelson (1984) Dopamine-containing amacrine cells of rhesus monkey retina parallel rods in spatial distribution. Brain Res. 322: 1-7.

Mariani, A. P., M. T. Caserta, and J. L. Barker (1986) Morphological and histochemical characterization of three types of dopamine-containing neurons in primary cultures of mouse and rat spinal cord. Brain Res. 376: 335-341.

Nguyen-Legros, J., C. Botteri, L. H. Phuc, A. Vigny, and M. Gay (1984) Morphology of primate's dopaminergic amacrine cells as revealed by $\mathrm{TH}$-like immunoreactivity on retinal flat-mounts. Brain Res. 295: 145-153.

O'Brien, D. R., and J. E. Dowling (1985) Dopamine regulation of GABA release from the intact goldfish retina. Brain Res. 360: 41-50.

Pickel, V. M., T. H. Joh, and D. J. Reis (1975) Immunohistochemical localization of tyrosine hydroxylase in brain by light and electron microscopy. Brain Res. 85: 295-300.

Polyak, S. L. (1957) Structure of the retina. In The Vertebrate Visual System, pp. 207-287, Chicago U. P., Chicago.

Pourcho, R. G. (1982) Dopaminergic amacrine cells in the cat retina. Brain Res. 252: 101-109.

Sternberger, L. A. (1986) Immunocytochemistry, 3rd Ed., Wiley, New York.

Teranishi, T., K. Negishi, and S. Kato (1983) Dopamine modulates S-potential amplitude and dye-coupling between external horizontal cells in carp retina. Nature 301: 243-246.

Venable, J. H., and R. Coggeshall (1965) A simplified lead citrate stain for use in electron microscopy. J. Cell Biol. 25: 407-408.

Watling, K. J., J. E. Dowling, and L. L. Iversen (1979) Dopamine receptors in the retina may all be linked to adenylate cyclase. Nature 281: $578-580$. 